\title{
Generalized Bell Numbers and Peirce Matrix via Pascal Matrix
}

\author{
Eunmi Choi \\ Department of Mathematics, HanNam Univ., Daejeon, Republic of Korea \\ Correspondence should be addressed to Eunmi Choi; emc@hnu.kr \\ Received 20 June 2018; Accepted 29 July 2018; Published 8 August 2018 \\ Academic Editor: Pentti Haukkanen
}

Copyright (c) 2018 Eunmi Choi. This is an open access article distributed under the Creative Commons Attribution License, which permits unrestricted use, distribution, and reproduction in any medium, provided the original work is properly cited.

With the Stirling matrix $S$ and the Pascal matrix $T$, we show that $T^{k} S(k \geq 0)$ satisfies a type of generalized Stirling recurrence. Then, by expressing the sum of components of each row of $T^{k} S$ as $k$-Bell number, we investigate properties of $k$-Bell numbers as well as $k$-Peirce matrix.

\section{Introduction}

The Stirling number $s_{m, n}$ of the second kind is the number of ways to partition an $m$ elements set into $n$ nonempty subsets for any $m, n \geq 0 . s_{m, n}$ can be expressed by $n ! s_{m, n}=\sum_{i=0}^{n}(-1)^{i}\left(\begin{array}{c}n \\ i\end{array}\right)(n-i)^{m}$, and the Stirling matrix $S=$ $\left[s_{m, n}\right](m, n \geq 0)$ satisfies the Stirling recurrence $s_{m, n}=$ $s_{m-1, n-1}+n s_{m-1, n}[1]$. Some researches including [2-4] were devoted to investigating the Stirling matrix $S$ with the Pascal matrix $T$ and binomial expressions. On the other hand, the sum $B_{m}=\sum_{k=0}^{m} s_{m, k}$ of numbers of the $m$ th row of $S$ is called a Bell number, so $\left\{B_{m} \mid m \geq 0\right\}=\{1,1,2,5,15, \cdots\}$ counts the number of partitions of an $m$ elements set. One effective way for generating Bell numbers is to use the Peirce matrix (often called Bell table or Aitken's array), on which Bell numbers appear along both borders [5-7].

A main purpose of work is to study $T^{k} S$ for $k \geq 1$. We show that $T^{k} S$ satisfies a kind of generalized Stirling recurrence, and then by expressing the sum of components of each row of $T^{k} S$ as a $k$-Bell number we investigate $k$-Bell numbers as well as $k$-Peirce matrix. We discuss recurrence rules of a $k$-Peirce matrix and then interrelationships between each $k$-Peirce matrices.

\section{Generalized $k$-Stirling Matrix and $k$-Bell Numbers}

Throughout the work, we write $T$ for the Pascal matrix and $r_{m}$ for the $m$ th row of $T$. And $M^{t r}$ means a be

transpose matrix of $M$. Let the Stirling matrix

$$
S=\left[\begin{array}{llllll}
1 & & & & \\
0 & 1 & & & \\
0 & 1 & 1 & & \\
0 & 1 & 3 & 1 & \\
0 & 1 & 7 & 6 & 1 \\
& \cdots & & &
\end{array}\right]
$$$$
\text { and } S^{*}=\left[\begin{array}{cccccc}
1 & & & & \\
1 & 1 & & & \\
1 & 3 & 1 & & \\
1 & 7 & 6 & 1 & \\
1 & 15 & 25 & 10 & 1 \\
& \ldots & & &
\end{array}\right] \text {. }
$$

Theorem 1. We have the following: 
(1) $S\left[\begin{array}{l}1 \\ 2 \\ 3 \\ \ldots\end{array}\right]=\left[\begin{array}{l}B_{1} \\ B_{2} \\ B_{3} \\ \ldots\end{array}\right]=S^{*}\left[\begin{array}{l}1 \\ 1 \\ 1 \\ \ldots\end{array}\right], S^{*}\left[\begin{array}{l}1 \\ 2 \\ 3 \\ \ldots\end{array}\right]=\left[\begin{array}{c}B_{2}-B_{1} \\ B_{3}-B_{2} \\ B_{4}-B_{3} \\ \ldots\end{array}\right]$ and $S^{*}\left[\begin{array}{c}2 \\ 3 \\ 4 \\ \cdots\end{array}\right]=\left[\begin{array}{l}B_{2} \\ B_{3} \\ B_{4} \\ \cdots\end{array}\right]$.

(2) $T\left[\begin{array}{l}B_{0} \\ B_{1} \\ B_{2} \\ \cdots\end{array}\right]=\left[\begin{array}{l}B_{1} \\ B_{2} \\ B_{3} \\ \cdots\end{array}\right]$ and $T\left[\begin{array}{c}B_{1} \\ B_{2} \\ B_{3} \\ \ldots\end{array}\right]=S^{*}\left[\begin{array}{c}1 \\ 2 \\ 3 \\ \cdots\end{array}\right]$.

Proof. The first two identities in (1) are easy to observe. And

$$
\begin{aligned}
S^{*}\left[\begin{array}{l}
2 \\
3 \\
4 \\
\ldots
\end{array}\right] & =S^{*}\left[\begin{array}{l}
1 \\
1 \\
1 \\
\ldots
\end{array}\right]+S^{*}\left[\begin{array}{c}
1 \\
2 \\
3 \\
\ldots
\end{array}\right] \\
& =\left[\begin{array}{c}
B_{1} \\
B_{2} \\
B_{3} \\
\ldots
\end{array}\right]+\left[\begin{array}{c}
B_{2}-B_{1} \\
B_{3}-B_{2} \\
B_{4}-B_{3} \\
\ldots
\end{array}\right]=\left[\begin{array}{c}
B_{2} \\
B_{3} \\
B_{4} \\
\ldots
\end{array}\right] .
\end{aligned}
$$

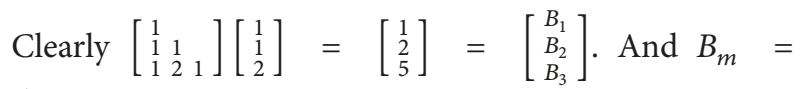
$\sum_{i=0}^{m-1}\left(\begin{array}{c}m-1 \\ i\end{array}\right) B_{i}$ in [8] proves $r_{m}\left(B_{0}, \cdots, B_{m-1}\right)^{t r}=B_{m}$ for $m \geq 1$, so we have $T\left[\begin{array}{c}B_{0} \\ B_{1} \\ B_{2} \\ \ldots\end{array}\right]=\left[\begin{array}{c}B_{1} \\ B_{2} \\ B_{3} \\ \ldots\end{array}\right]$. Moreover since

$$
\begin{aligned}
r_{m+1}= & \left(\left(\begin{array}{c}
m-1 \\
0
\end{array}\right), \cdots,\left(\begin{array}{c}
m-1 \\
m-1
\end{array}\right), 0\right) \\
& +\left(0,\left(\begin{array}{c}
m-1 \\
0
\end{array}\right), \cdots,\left(\begin{array}{c}
m-1 \\
m-1
\end{array}\right)\right) \\
= & \left(r_{m} ; 0\right)+\left(0 ; r_{m}\right),
\end{aligned}
$$

it follows that

$$
\begin{aligned}
B_{m+1}= & r_{m+1}\left(B_{0}, B_{1}, \cdots, B_{m}\right)^{t r} \\
= & \left(r_{m} ; 0\right)\left(B_{0}, B_{1}, \cdots, B_{m}\right)^{t r} \\
& +\left(0 ; r_{m}\right)\left(B_{0}, B_{1}, \cdots, B_{m}\right)^{t r} \\
= & r_{m}\left(B_{0}, \cdots, B_{m-1}\right)^{t r}+r_{m}\left(B_{1}, \cdots, B_{m}\right)^{t r} \\
= & B_{m}+r_{m}\left(B_{1}, \cdots, B_{m}\right)^{t r} .
\end{aligned}
$$

So $r_{m}\left(B_{1}, \cdots, B_{m}\right)^{t r}=B_{m+1}-B_{m}$ and $T\left[\begin{array}{c}B_{1} \\ B_{2} \\ B_{3} \\ \cdots\end{array}\right]=\left[\begin{array}{c}B_{2}-B_{1} \\ B_{3}-B_{2} \\ B_{4}-B_{3} \\ \cdots\end{array}\right]=$ $S^{*}\left[\begin{array}{l}1 \\ 2 \\ 3 \\ \cdots\end{array}\right]$.

Hence the next corollary follows immediately.

Corollary $2\left(T S=S^{*}\right)$. As a generalization of $S^{*}=T S$, we shall consider $T^{k} S$ for $k>0$. Write $S^{(k)}=T^{k} S=\left[s_{m, n}^{(k)}\right]$ for $m, n \geq 0$, and let $S^{(0)}=S$. Clearly $S^{(1)}=S^{*}$.
Theorem 3. $S^{(k)}$ satisfies the recurrence $s_{m+1, n+1}^{(k)}=s_{m, n}^{(k)}+(n+$ k) $s_{m, n+1}^{(k)}$.

Proof. $S^{(1)}=\left[s_{m, n}^{(1)}\right]=S^{*}$ in Corollary 2 shows $s_{m+1, n+1}^{(1)}=$

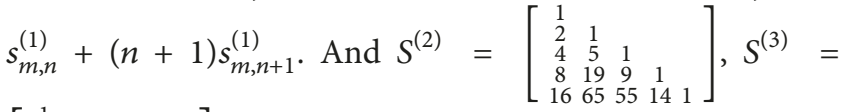
$\left[\begin{array}{ccccc}1 & & & & \\ 3 & 1 & & \\ 9 & 7 & 1 & \\ 27 & 37 & 12 & 1 \\ 81 & 175 & 97 & 18 & 1\end{array}\right]$ give identities $55=19+(4) 9$ and $97=$ $37+(5) 12$, and it is easy to observe

$$
s_{m+1, n+1}^{(k)}=s_{m, n}^{(k)}+(n+k) s_{m, n+1}^{(k)}
$$

for $1 \leq n, m \leq 5 ; k=2,3$. Now we assume $s_{m+1, n+1}^{(k)}=s_{m, n}^{(k)}+$ $(n+k) s_{m, n+1}^{(k)}$ for some $k, m$. Since

$$
\left[s_{m, n}^{(k+1)}\right]=S^{(k+1)}=T^{k+1} S=T T^{k} S=T S^{(k)},
$$

any $(m, n)$ th component $s_{m, n}^{(k+1)}$ comes from the $m$ th row $r_{m}$ of $T$ and the $n$th column of $S^{(k)}$. So

$$
\begin{aligned}
& s_{m, n}^{(k+1)}=(\underbrace{\left(\begin{array}{c}
m-1 \\
0
\end{array}\right), \cdots,\left(\begin{array}{c}
m-1 \\
n-2
\end{array}\right)}_{n-1},\left(\begin{array}{c}
m-1 \\
n-1
\end{array}\right), \\
& \left.\left(\begin{array}{c}
m-1 \\
n
\end{array}\right), \cdots,\left(\begin{array}{c}
m-1 \\
m-2
\end{array}\right),\left(\begin{array}{c}
m-1 \\
m-1
\end{array}\right)\right) \cdot(\underbrace{0, \cdots, 0}_{n-1}, s_{n, n}^{(k)}, \\
& \left.s_{n+1, n}^{(k)}, \cdots, s_{m-1, n}^{(k)}, s_{m, n}^{(k)}\right)^{t r}=\left(\begin{array}{c}
m-1 \\
n-1
\end{array}\right) s_{n, n}^{(k)} \\
& +\left(\begin{array}{c}
m-1 \\
n
\end{array}\right) s_{n+1, n}^{(k)}+\cdots+\left(\begin{array}{c}
m-1 \\
m-2
\end{array}\right) s_{m-1, n}^{(k)} \\
& +\left(\begin{array}{c}
m-1 \\
m-1
\end{array}\right) s_{m, n}^{(k)}
\end{aligned}
$$

and

$$
\begin{aligned}
& s_{m, n+1}^{(k+1)}=(\underbrace{\left(\begin{array}{c}
m-1 \\
0
\end{array}\right), \cdots,\left(\begin{array}{c}
m-1 \\
n-1
\end{array}\right)}_{n},\left(\begin{array}{c}
m-1 \\
n
\end{array}\right), \\
& \left.\left(\begin{array}{c}
m-1 \\
n+1
\end{array}\right), \cdots,\left(\begin{array}{c}
m-1 \\
m-2
\end{array}\right),\left(\begin{array}{c}
m-1 \\
m-1
\end{array}\right)\right) \cdot(\underbrace{0, \cdots, 0}_{n}, \\
& \left.s_{n+1, n+1}^{(k)}, s_{n+2, n+1}^{(k)}, \cdots, s_{m-1, n+1}^{(k)}, s_{m, n+1}^{(k)}\right)^{t r} \\
& =\left(\begin{array}{c}
m-1 \\
n
\end{array}\right) s_{n+1, n+1}^{(k)}+\left(\begin{array}{c}
m-1 \\
n+1
\end{array}\right) s_{n+2, n+1}^{(k)}+\cdots \\
& +\left(\begin{array}{l}
m-1 \\
m-2
\end{array}\right) s_{m-1, n+1}^{(k)}+s_{m, n+1}^{(k)} .
\end{aligned}
$$


International Journal of Mathematics and Mathematical Sciences

3

Therefore we have

$$
\begin{aligned}
& s_{m, n}^{(k+1)}+(n+k+1) s_{m, n+1}^{(k+1)}=\left[\left(\begin{array}{c}
m-1 \\
n-1
\end{array}\right) s_{n, n}^{(k)}\right. \\
& \left.+\left(\begin{array}{c}
m-1 \\
n
\end{array}\right) s_{n+1, n}^{(k)}+\cdots+\left(\begin{array}{c}
m-1 \\
m-2
\end{array}\right) s_{m-1, n}^{(k)}+s_{m, n}^{(k)}\right] \\
& +(n+k+1)\left[\left(\begin{array}{c}
m-1 \\
n
\end{array}\right) s_{n+1, n+1}^{(k)}+\cdots\right. \\
& \left.+\left(\begin{array}{c}
m-1 \\
m-2
\end{array}\right) s_{m-1, n+1}^{(k)}+s_{m, n+1}^{(k)}\right]=\left[\left(\begin{array}{c}
m-1 \\
n-1
\end{array}\right) s_{n, n}^{(k)}\right. \\
& \left.+\left(\begin{array}{c}
m-1 \\
n
\end{array}\right) s_{n+1, n}^{(k)}+\cdots+\left(\begin{array}{c}
m-1 \\
m-2
\end{array}\right) s_{m-1, n}^{(k)}+s_{m, n}^{(k)}\right] \\
& +\left[\left(\begin{array}{c}
m-1 \\
n
\end{array}\right)(n+k) s_{n+1, n+1}^{(k)}+\cdots\right. \\
& \left.+\left(\begin{array}{c}
m-1 \\
m-2
\end{array}\right)(n+k) s_{m-1, n+1}^{(k)}+(n+k) s_{m, n+1}^{(k)}\right] \\
& +\left[\left(\begin{array}{c}
m-1 \\
n
\end{array}\right) s_{n+1, n+1}^{(k)}+\cdots+\left(\begin{array}{c}
m-1 \\
m-2
\end{array}\right) s_{m-1, n+1}^{(k)}\right. \\
& \left.+s_{m, n+1}^{(k)}\right]=\left(\begin{array}{c}
m-1 \\
n-1
\end{array}\right) s_{n, n}^{(k)}+\left(\begin{array}{c}
m-1 \\
n
\end{array}\right)\left[s_{n+1, n}^{(k)}\right. \\
& \left.+(n+k) s_{n+1, n+1}^{(k)}\right]+\cdots+\left(\begin{array}{l}
m-1 \\
m-2
\end{array}\right)\left[s_{m-1, n}^{(k)}\right. \\
& \left.+(n+k) s_{m-1, n+1}^{(k)}\right]+\left[s_{m, n}^{(k)}+(n+k) s_{m, n+1}^{(k)}\right] \\
& +\left[\left(\begin{array}{c}
m-1 \\
n
\end{array}\right) s_{n+1, n+1}^{(k)}+\cdots+\left(\begin{array}{c}
m-1 \\
m-2
\end{array}\right) s_{m-1, n+1}^{(k)}\right. \\
& \left.+s_{m, n+1}^{(k)}\right]=\left(\begin{array}{c}
m-1 \\
n-1
\end{array}\right) s_{n, n}^{(k)}+\left(\begin{array}{c}
m-1 \\
n
\end{array}\right) s_{n+2, n+1}^{(k)}+\cdots \\
& +\left(\begin{array}{c}
m-1 \\
m-2
\end{array}\right) s_{m, n+1}^{(k)}+s_{m+1, n+1}^{(k)}+\left[\left(\begin{array}{c}
m-1 \\
n
\end{array}\right) s_{n+1, n+1}^{(k)}\right. \\
& \left.+\cdots+\left(\begin{array}{l}
m-1 \\
m-2
\end{array}\right) s_{m-1, n+1}^{(k)}+s_{m, n+1}^{(k)}\right]
\end{aligned}
$$

by the induction hypothesis on $S^{(k)}$. But since $s_{i, i}^{(k)}=1$ for all $i$, it follows immediately from Pascal's rule that

$$
\begin{aligned}
& s_{m, n}^{(k+1)}+(n+k+1) s_{m, n+1}^{(k+1)} \\
& =\left[\left(\begin{array}{c}
m-1 \\
n-1
\end{array}\right)+\left(\begin{array}{c}
m-1 \\
n
\end{array}\right)\right] s_{n, n}^{(k)} \\
& +\left[\left(\begin{array}{c}
m-1 \\
n
\end{array}\right)+\left(\begin{array}{c}
m-1 \\
n+1
\end{array}\right)\right] s_{n+2, n+1}^{(k)}+\cdots \\
& +\left[\left(\begin{array}{l}
m-1 \\
m-2
\end{array}\right)+1\right] s_{m, n+1}^{(k)}+s_{m+1, n+1}^{(k)} \\
& =\left(\begin{array}{c}
m \\
n
\end{array}\right) s_{n, n}^{(k)}+\left(\begin{array}{c}
m \\
n+1
\end{array}\right) s_{n+2, n+1}^{(k)}+\cdots \\
& +\left(\begin{array}{c}
m \\
m-1
\end{array}\right) s_{m, n+1}^{(k)}+s_{m+1, n+1}^{(k)} \\
& =\left(\begin{array}{c}
m \\
n
\end{array}\right) s_{n+1, n+1}^{(k)}+\left(\begin{array}{c}
m \\
n+1
\end{array}\right) s_{n+2, n+1}^{(k)}+\cdots \\
& +\left(\begin{array}{c}
m \\
m-1
\end{array}\right) s_{m, n+1}^{(k)}+s_{m+1, n+1}^{(k)} \\
& =(\underbrace{\left(\begin{array}{c}
m \\
0
\end{array}\right), \cdots,\left(\begin{array}{c}
m \\
n-1
\end{array}\right)}_{n},\left(\begin{array}{l}
m \\
n
\end{array}\right), \cdots,\left(\begin{array}{c}
m \\
m-1
\end{array}\right), 1) \\
& \cdot(\underbrace{0, \cdots, 0}_{n}, s_{n+1, n+1}^{(k)}, \cdots, s_{m+1, n+1}^{(k)})^{t r}=s_{m+1, n+1}^{(k+1)} \text {. }
\end{aligned}
$$


Obviously $B_{i}^{(0)}=B_{i}$ the (original) Bell numbers, and $B_{i}^{(1)}=B_{i+1}^{(0)}$, so $B_{i}^{(2)}=B_{i+1}^{(1)}-B_{i}^{(1)}=B_{i+2}^{(0)}-B_{i+1}^{(0)}(i \geq 0)$ by Theorem 1. Note that $B^{(2)}$ is listed in A005493-OEIS as the numbers of partitions with a distinguished block. And $B^{(k)}(3 \leq k \leq 5)$ are coefficients of $\exp (k x+\exp (x)-1)$ (A005494, A045379, and A196834). On the other hand, the $k$-Bell numbers $B^{(k)}$ were studied in [9] by using certain binomial expressions $B_{m}=\sum_{i=0}^{m}\left\{\begin{array}{c}m \\ i\end{array}\right\}$ and $B_{m}^{(k)}=\sum_{i=0}^{m}\left\{\begin{array}{c}m+k \\ i+k\end{array}\right\}$. A key feature of our study is to have these $k$-Bell numbers from multiplications of $T$ and $S$ explicitly. Hence it enables us to find relations between $k$-Bell numbers $B^{(k)}$ and $B^{(k+1)}$, as follows.

Theorem 4. $S^{(k)}\left[\begin{array}{c}1 \\ 1 \\ 1 \\ \ldots\end{array}\right]=T\left[\begin{array}{c}B_{0}^{(k-1)} \\ B_{1}^{(k-1)} \\ B_{2 . .}^{(k-1)}\end{array}\right]$ and $S^{(k)}\left[\begin{array}{c}1 \\ 2 \\ 3 \\ \ldots\end{array}\right]=\left[\begin{array}{c}B_{0}^{(k+1)} \\ B_{1}^{(k+1)} \\ B_{2 . .}^{(k+1)}\end{array}\right]$. Proof. Since $S^{(k)}=T^{k} S=T S^{(k-1)}$, it is clear that

$$
\left[\begin{array}{c}
B_{0}^{(k)} \\
B_{1}^{(k)} \\
B_{2}^{(k)} \\
\ldots
\end{array}\right]=S^{(k)}\left[\begin{array}{c}
1 \\
1 \\
1 \\
\ldots
\end{array}\right]=T S^{(k-1)}\left[\begin{array}{c}
1 \\
1 \\
1 \\
\ldots
\end{array}\right]=T\left[\begin{array}{c}
B_{0}^{(k-1)} \\
B_{1}^{(k-1)} \\
B_{2}^{(k-1)} \\
\ldots
\end{array}\right] .
$$

And, due to Theorem 1, we have

$$
\begin{aligned}
S^{(0)}\left[\begin{array}{c}
1 \\
2 \\
3 \\
\ldots
\end{array}\right] & =\left[\begin{array}{c}
B_{1}^{(0)} \\
B_{2}^{(0)} \\
B_{3}^{(0)} \\
\ldots
\end{array}\right]=\left[\begin{array}{c}
B_{0}^{(1)} \\
B_{1}^{(1)} \\
B_{2}^{(1)} \\
\ldots
\end{array}\right] \\
\text { and } S^{(1)}\left[\begin{array}{c}
1 \\
2 \\
3 \\
\ldots
\end{array}\right] & =\left[\begin{array}{c}
B_{2}^{(0)}-B_{1}^{(0)} \\
B_{3}^{(0)}-B_{2}^{(0)} \\
B_{4}^{(0)}-B_{3}^{(0)} \\
\ldots
\end{array}\right]=\left[\begin{array}{c}
B_{0}^{(2)} \\
B_{1}^{(2)} \\
B_{2}^{(2)} \\
\ldots
\end{array}\right] .
\end{aligned}
$$

So if we assume $S^{(k-1)}\left[\begin{array}{c}1 \\ 2 \\ 3 \\ \cdots\end{array}\right]=\left[\begin{array}{c}B_{0}^{(k)} \\ B_{1}^{(k)} \\ B_{2}^{(k)}\end{array}\right]$ for some $k$ then we have

$$
S^{(k)}\left[\begin{array}{c}
1 \\
2 \\
3 \\
\ldots
\end{array}\right]=T S^{(k-1)}\left[\begin{array}{c}
1 \\
2 \\
3 \\
\ldots
\end{array}\right]=T\left[\begin{array}{c}
B_{0}^{(k)} \\
B_{1}^{(k)} \\
B_{2}^{(k)} \\
\ldots
\end{array}\right]=\left[\begin{array}{c}
B_{0}^{(k+1)} \\
B_{1}^{(k+1)} \\
B_{2}^{(k+1)} \\
\ldots
\end{array}\right] .
$$

Theorem 4 says $T\left[\begin{array}{l}B_{0} \\ B_{1} \\ B_{2} \\ \ldots\end{array}\right]=\left[\begin{array}{l}B_{1} \\ B_{2} \\ B_{3} \\ \cdots\end{array}\right]$, so $T\left[\begin{array}{l}B_{1} \\ B_{2} \\ B_{3} \\ \ldots\end{array}\right]=$ $T^{2}\left[\begin{array}{c}B_{0} \\ B_{1} \\ B_{2} \\ \ldots\end{array}\right]$. Thus the expression $r_{m}\left(B_{1}, \cdots, B_{m}\right)^{t r}$ in the proof of Theorem 1, which is the multiplication of the $m$ th row of $T$ with $\left(B_{1}, \cdots, B_{m}\right)^{t r}$, is equal to multiplication of the $m$ th row of $T^{2}$ with $\left(B_{0}, \cdots, B_{m-1}\right)^{t r}$. Note that $T^{2}$ equals the arithmetic table $T^{(2 x+1)}$ of the polynomial $(2 x+1)^{n}$, while Pascal's table $T$ is the arithmetic table of $(x+1)^{n}$. Hence if we let $r_{m}^{(2 x+1)}$ be the $m$ th row of $T^{(2 x+1)}=\left[\begin{array}{llll}1 & 1 & \\ 2 & 1 & 1 \\ 8 & 4 & 6 & 1\end{array}\right]$ of $(2 x+1)^{n}$ then

$$
\begin{aligned}
& B_{m+1}-B_{m}=\sum_{i=0}^{m}\left(\begin{array}{c}
m-1 \\
i
\end{array}\right) B_{i+1} \\
& =\left(\left(\begin{array}{c}
m-1 \\
0
\end{array}\right),\left(\begin{array}{c}
m-1 \\
1
\end{array}\right), \cdots,\left(\begin{array}{c}
m-1 \\
m-1
\end{array}\right)\right) \\
& \cdot\left(B_{1}, \cdots, B_{m}\right)^{t r}=r_{m}^{(2 x+1)}\left(B_{0}, \cdots, B_{m-1}\right)^{t r},
\end{aligned}
$$

which implies $T^{2}\left[\begin{array}{c}B_{0} \\ B_{2} \\ \ldots\end{array}\right]=\left[\begin{array}{c}B_{2}-B_{1} \\ B_{3}-B_{2} \\ \ldots .\end{array}\right]=\left[\begin{array}{c}B_{0}^{(2)} \\ B_{1}^{(2)} \\ \ldots\end{array}\right]=S^{(2)}\left[\begin{array}{l}1 \\ 1 \\ \ldots\end{array}\right]$. Thus, owing to Theorem 4 , it is clear to have generalizations to $k$ Bell numbers $(k \geq 0)$ that

$$
\begin{aligned}
T^{u}\left[\begin{array}{c}
B_{0}^{(k)} \\
B_{1}^{(k)} \\
B_{2}^{(k)} \\
\ldots
\end{array}\right] & =T^{u} S^{(k)}\left[\begin{array}{c}
1 \\
1 \\
1 \\
\ldots
\end{array}\right]=S^{(u+k)}\left[\begin{array}{c}
1 \\
1 \\
1 \\
\ldots
\end{array}\right] \\
& =\left[\begin{array}{c}
B_{0}^{(u+k)} \\
B_{1}^{(u+k)} \\
B_{2}^{(u+k)} \\
\cdots
\end{array}\right] .
\end{aligned}
$$

\section{Matrix of General Bell Numbers}

With all the $j$-Bell numbers $B^{(j)}(j \geq 1)$, we make a table $\widehat{G B}$ in which each $j$ th column is composed of $j$-Bell numbers. We call it a matrix of general Bell numbers and denote it by $\widehat{G B}=\left[b_{i, j}\right](i, j \geq 1)$.

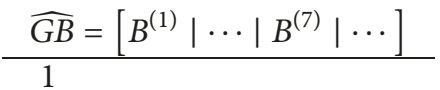

$$
\begin{aligned}
& 21 \\
& \begin{array}{lll}
5 & 3 & 1
\end{array} \\
& \begin{array}{llll}
15 & 10 & 4 & 1
\end{array} \\
& \begin{array}{lllll}
52 & 37 & 17 & 5 & 1
\end{array} \\
& \begin{array}{llllll}
203 & 151 & 77 & 26 & 6 & 1
\end{array} \\
& \begin{array}{lllllll}
877 & 674 & 372 & 141 & 37 & 7 & 1
\end{array}
\end{aligned}
$$

Theorem 5. The entries of the $(j+1)$ th column in $\widehat{G B}=\left[b_{i, j}\right]$ satisfy $b_{i, j+1}=b_{i, j}-j b_{i-1, j}$. So $B_{i}^{(j+1)}=B_{i+1}^{(j)}-j B_{i}^{(j)}$.

Proof. Observe that each $j$ th column $(2 \leq j \leq 5)$ satisfies the following: 


\begin{tabular}{c|c|c|c} 
2nd col & 3rd col & 4 th col & 5 th col \\
\hline $2-1=1$ & $3-(2) 1=1$ & $4-(3) 1=1$ & $5-(4) 1=1$ \\
$5-2=3$ & $10-(2) 3=4$ & $17-(3) 4=5$ & $26-(4) 5=6$ \\
$15-5=10$ & $37-(2) 10=17$ & $77-(3) 17=26$ & $141-(4) 26=37$ \\
$52-15=37$ & $151-(2) 37=77$ & $372-(3) 77=141$ & $799-(4) 141=235$
\end{tabular}

at

Suppose $b_{i, j+1}=b_{i, j}-j b_{i-1, j}$ for some $i, j>1$. By looking

$$
\begin{aligned}
{\left[b_{i, j}\right] } & =\left[\begin{array}{llll}
b_{1,1} & & \\
b_{2,1} & b_{2,2} & & \\
b_{3,1} & b_{3,2} & b_{3,3} & \\
b_{4,1} & b_{4,2} & b_{4,3} & b_{4,4} \\
\cdots & & &
\end{array}\right] \\
= & {\left[\begin{array}{llll}
B_{0}^{(1)} & & & \\
B_{1}^{(1)} & B_{0}^{(2)} & & \\
B_{2}^{(1)} & B_{1}^{(2)} & B_{0}^{(3)} & \\
B_{3}^{(1)} & B_{2}^{(2)} & B_{1}^{(3)} & B_{0}^{(4)} \\
\cdots & &
\end{array}\right] }
\end{aligned}
$$

it is enough to prove the following two cases.

(i) For any $i \geq 0$, assume $B_{i}^{(j)}=B_{i+1}^{(j-1)}-(j-1) B_{i}^{(j-1)}$ in some $j$ th column and show $B_{i}^{(j+1)}=B_{i+1}^{(j)}-j B_{i}^{(j)}$ is true at $(j+1)$ th column.

(ii) For any $j \geq 1$, assume $B_{t-1}^{(j)}=B_{t}^{(j-1)}-(j-1) B_{t-1}^{(j-1)}$ at every $t$ th row $(1 \leq t \leq i)$ and show $B_{i}^{(j)}=B_{i+1}^{(j-1)}-(j-1) B_{i}^{(j-1)}$ is true at $(i+1)$ th row.

Using the $i$ th row $r_{i}$ of $T$, the induction hypothesis in (i) with (16) implies

$$
\begin{aligned}
& B_{i}^{(j+1)}=r_{i+1}\left(B_{0}^{(j)}, B_{1}^{(j)}, \cdots B_{i}^{(j)}\right)^{t r}=r_{i+1}\left(B_{1}^{(j-1)}\right. \\
& \left.-(j-1) B_{0}^{(j-1)}, \cdots, B_{i+1}^{(j-1)}-(j-1) B_{i}^{(j-1)}\right)^{t r} \\
& =r_{i+1}\left(B_{1}^{(j-1)}, \cdots, B_{i+1}^{(j-1)}\right)^{t r}-(j-1) \\
& \cdot r_{i+1}\left(B_{0}^{(j-1)}, \cdots, B_{i}^{(j-1)}\right)^{t r}=\left[\left(\begin{array}{l}
i \\
0
\end{array}\right) B_{1}^{(j-1)}\right. \\
& \left.+\left(\begin{array}{l}
i \\
1
\end{array}\right) B_{2}^{(j-1)}+\cdots+\left(\begin{array}{l}
i \\
i
\end{array}\right) B_{i+1}^{(j-1)}\right]-(j-1) \\
& \cdot\left[\left(\begin{array}{l}
i \\
0
\end{array}\right) B_{0}^{(j-1)}+\left(\begin{array}{l}
i \\
1
\end{array}\right) B_{1}^{(j-1)}+\cdots+\left(\begin{array}{l}
i \\
i
\end{array}\right) B_{i}^{(j-1)}\right] \\
& =\left(-(j-1)\left(\begin{array}{l}
i \\
0
\end{array}\right),\left(\begin{array}{l}
i \\
0
\end{array}\right)\right.
\end{aligned}
$$

$$
\begin{aligned}
& \left.-(j-1)\left(\begin{array}{c}
i \\
1
\end{array}\right), \cdots,\left(\begin{array}{c}
i \\
i-1
\end{array}\right)-(j-1)\left(\begin{array}{l}
i \\
i
\end{array}\right),\left(\begin{array}{l}
i \\
i
\end{array}\right)\right) \\
& \cdot\left(B_{0}^{(j-1)}, B_{1}^{(j-1)}, \cdots, B_{i+1}^{(j-1)}\right)^{t r} .
\end{aligned}
$$

On the other hand, we also have

$$
\begin{aligned}
& B_{i+1}^{(j)}-j B_{i}^{(j)}=r_{i+2}\left(B_{0}^{(j-1)}, B_{1}^{(j-1)}, \cdots, B_{i+1}^{(j-1)}\right)^{t r} \\
& -j r_{i+1}\left(B_{0}^{(j-1)}, B_{1}^{(j-1)}, \cdots, B_{i}^{(j-1)}\right)^{t r} \\
& =\left[\left(\begin{array}{c}
i+1 \\
0
\end{array}\right) B_{0}^{(j-1)}+\left(\begin{array}{c}
i+1 \\
1
\end{array}\right) B_{1}^{(j-1)}\right. \\
& \left.+\left(\begin{array}{c}
i+1 \\
2
\end{array}\right) B_{2}^{(j-1)}+\cdots+\left(\begin{array}{c}
i+1 \\
i+1
\end{array}\right) B_{i+1}^{(j-1)}\right] \\
& -j\left[\left(\begin{array}{l}
i \\
0
\end{array}\right) B_{0}^{(j-1)}+\left(\begin{array}{l}
i \\
1
\end{array}\right) B_{1}^{(j-1)}+\cdots+\left(\begin{array}{l}
i \\
i
\end{array}\right) B_{i}^{(j-1)}\right] \\
& =\left(\left(\begin{array}{c}
i+1 \\
0
\end{array}\right)-j\left(\begin{array}{l}
i \\
0
\end{array}\right),\left(\begin{array}{c}
i+1 \\
1
\end{array}\right)-j\left(\begin{array}{l}
i \\
1
\end{array}\right),\left(\begin{array}{c}
i+1 \\
2
\end{array}\right)\right. \\
& \left.-j\left(\begin{array}{l}
i \\
2
\end{array}\right), \cdots,\left(\begin{array}{c}
i+1 \\
i
\end{array}\right)-j\left(\begin{array}{l}
i \\
i
\end{array}\right),\left(\begin{array}{c}
\mathrm{i}+1 \\
i+1
\end{array}\right)\right) \\
& \cdot\left(B_{0}^{(j-1)}, \cdots, B_{i}^{(j-1)}, B_{i+1}^{(j-1)}\right)^{t r} \\
& =\left(-(j-1)\left(\begin{array}{l}
i \\
0
\end{array}\right),\left(\begin{array}{l}
i \\
0
\end{array}\right)\right. \\
& \left.-(j-1)\left(\begin{array}{l}
i \\
1
\end{array}\right), \cdots,\left(\begin{array}{c}
i \\
i-1
\end{array}\right)-(j-1)\left(\begin{array}{l}
i \\
i
\end{array}\right),\left(\begin{array}{l}
i \\
i
\end{array}\right)\right) \\
& \cdot\left(B_{0}^{(j-1)}, B_{1}^{(j-1)}, \cdots, B_{i+1}^{(j-1)}\right)^{t r},
\end{aligned}
$$

for $\left(\begin{array}{c}i+1 \\ u\end{array}\right)-j\left(\begin{array}{c}i \\ u\end{array}\right)=\left(\begin{array}{c}i \\ u-1\end{array}\right)+\left(\begin{array}{l}i \\ u\end{array}\right)-j\left(\begin{array}{l}i \\ u\end{array}\right)=\left(\begin{array}{c}i \\ u-1\end{array}\right)-(j-1)+\left(\begin{array}{l}i \\ u\end{array}\right)$ for all $0 \leq u \leq i$. Hence identities (20) and (21) prove (i).

Now, for (ii), the induction hypothesis $B_{t}^{(j-1)}+(j-$ 2) $B_{t}^{(j-2)}=B_{t+1}^{(j-2)}(1 \leq t \leq i)$ in (ii) with again (16) implies

$$
\begin{aligned}
B_{i}^{(j)} & +(j-1) B_{i}^{(j-1)}=B_{i}^{(j)}+(j-2) B_{i}^{(j-1)}+B_{i}^{(j-1)} \\
& =r_{i+1}\left(B_{0}^{(j-1)}, \cdots, B_{i}^{(j-1)}\right)^{t r}+(j-2)
\end{aligned}
$$




$$
\begin{aligned}
& \cdot r_{i+1}\left(B_{0}^{(j-2)}, \cdots, B_{i}^{(j-2)}\right)^{t r} \\
& +r_{i+1}\left(B_{0}^{(j-2)}, \cdots, B_{i}^{(j-2)}\right)^{t r}=r_{i-1}\left(B_{0}^{(j-1)}\right. \\
& \left.+(j-2) B_{0}^{(j-2)}, \cdots, B_{i}^{(j-1)}+(j-2) B_{i}^{(j-2)}\right)^{t r} \\
& +r_{i+1}\left(B_{0}^{(j-2)}, \cdots, B_{i}^{(j-2)}\right)^{t r} \\
& =r_{i+1}\left(B_{1}^{(j-2)}, \cdots, B_{i+1}^{(j-2)}\right)^{t r} \\
& +r_{i+1}\left(B_{0}^{(j-2)}, \cdots, B_{i}^{(j-2)}\right)^{t r}
\end{aligned}
$$

\section{Moreover}

$$
\begin{aligned}
& B_{i+1}^{(j-1)}=r_{i+2}\left(B_{0}^{(j-2)}, B_{1}^{(j-2)}, \cdots, B_{i+1}^{(j-2)}\right)^{t r} \\
& =\left[\left(0 ; r_{i+1}\right)+\left(r_{i+1} ; 0\right)\right]\left(B_{0}^{(j-2)}, B_{1}^{(j-2)}, \cdots, B_{i+1}^{(j-2)}\right)^{t r} \\
& =r_{i+1}\left(B_{1}^{(j-2)}, \cdots, B_{i+1}^{(j-2)}\right)^{t r} \\
& \quad+r_{i+1}\left(B_{0}^{(j-2)}, \cdots, B_{i}^{(j-2)}\right)^{t r}
\end{aligned}
$$

Then we complete the proof by comparing (22) and (23).

Therefore Theorem 5 with (16) shows

$$
\begin{aligned}
T^{u}\left[\begin{array}{c}
B_{0}^{(k)} \\
B_{1}^{(k)} \\
B_{2}^{(k)} \\
\cdots
\end{array}\right] & {\left[\begin{array}{c}
B_{0}^{(u+k)} \\
B_{1}^{(u+k)} \\
B_{2}^{(u+k)} \\
\cdots
\end{array}\right] } \\
& =\left[\begin{array}{c}
B_{1}^{(u+k-1)}-(k+u-1) B_{0}^{(u+k-1)} \\
B_{2}^{(u+k-1)}-(k+u-1) B_{1}^{(u+k-1)} \\
B_{3}^{(u+k-1)}-(k+u-1) B_{2}^{(u+k-1)} \\
\cdots
\end{array}\right] .
\end{aligned}
$$

As an example, the 6-Bell numbers $B^{(6)}=\left\{B_{i}^{(6)}\right\}=$ $\{1,7,50,365,2727, \cdots\}$ and then 7 -Bell numbers $B^{(7)}=$ $\left\{B_{i}^{(7)}\right\}=\{1,8,65,537,4516, \cdots\}$ are obtained sequentially from the 5-Bell numbers.

\begin{tabular}{c|c|c} 
5th col. & 6 th col. & 7 th col. \\
\hline 6 & $6-(5) 1=1$ & $7-(6) 1=1$ \\
37 & $37-(5) 6=7$ & $50-(6) 7=8$ \\
235 & $235-(5) 37=50$ & $365-(6) 50=65$ \\
1540 & $1540-(5) 235=365$ & $2727-(6) 365=537$
\end{tabular}

The next theorem gives another way to have $k$-Bell numbers $B^{(k)}$. Let $[0]_{j}$ denote the $j$-tuple $\{0, \cdots, 0\}$.

Theorem 6. Let $X_{j}=\left([0]_{j-1} ; j, j+1, \cdots, n\right)^{\text {tr }}$ and $\rho_{n}=$ $\left(b_{n, 1}, \cdots, b_{n, n}\right)$ be the $n$th row of $\widehat{G B}$. Then $\rho_{n} X_{j}+1=b_{n+1, j}$ for any $1 \leq j \leq n$.
Proof. Clearly $\widehat{G B} X_{1}+1=\widehat{G B}\left[\begin{array}{l}1 \\ 2 \\ 3 \\ \ldots\end{array}\right]+1=\left[\begin{array}{c}2 \\ 5 \\ 15 \\ \ldots\end{array}\right]$ and $\widehat{G B} X_{2}+1=$ $\widehat{G B}\left[\begin{array}{c}0 \\ 2 \\ 3 \\ \ldots\end{array}\right]+1=\left[\begin{array}{c}1 \\ 3 \\ 10 \\ \ldots\end{array}\right]$. Similarly $\widehat{G B} X_{j}+1(j=3,4)$ equals $\left[\begin{array}{c}1 \\ 1 \\ 4 \\ \ldots\end{array}\right]$ and $\left[\begin{array}{l}1 \\ 1 \\ 1 \\ \ldots\end{array}\right]$. Thus, for instance, when $j=4$, with $n$th row $\rho_{n}$ of $\widehat{G B}$, we have $\rho_{3} X_{4}+1=d_{4,4}=1, \rho_{4} X_{4}+1=d_{5,4}=5$, $\rho_{5} X_{4}+1=d_{6,4}=26, \rho_{6} X_{4}+1=d_{7,4}=141$, and so on. Hence we can say that $\rho_{n} X_{4}+1=b_{n+1,4}$ for $n \geq 3$.

Now assume $\rho_{n} X_{j}+1=b_{n+1, j}-1$ for $n \geq j-1$. Then since $X_{j+1}=X_{j}-\left([0]_{j-1} ; j, 0, \cdots, 0\right)^{t r}$, Theorem 5 implies

$$
\begin{aligned}
\rho_{n} X_{j+1} & =\rho_{n} X_{j}-\rho_{n}\left([0]_{j-1} ; j, 0, \cdots, 0\right)^{t r} \\
& =b_{n+1, j}-1-j b_{n, j}=b_{n+1, j+1}-1 .
\end{aligned}
$$

Theorem 6 can be restated in terms of $k$-Bell numbers.

Corollary 7. $\left(B_{n}^{(1)}, B_{n-1}^{(2)}, \cdots, B_{1}^{(n)}\right)\left([0]_{j-1} ; j, j+1, \cdots, n\right)^{\operatorname{tr}}=$ $B_{n-j+2}^{(j)}-1$.

Hence $\widehat{G B}(1,2, \cdots, n)^{t r}=\left(B_{n+1}^{(1)}, \cdots, B_{n+1}^{(n)}\right)^{t r}$ and $\widehat{G B}\left([0]_{j-1} ; j, \cdots, n\right)^{t r}=\left([0]_{j-1} ; B_{n+1}^{(j)}, \cdots, B_{n+1}^{(n)}\right)^{t r}$. Precisely, with $\rho_{6}=(203,151,77,26,6,1)$, we have $\rho_{6}(0,0,0,0,0,6)^{t r}+$ $1=7, \rho_{6}(0,0,0,0,5,6)^{t r}+1=37, \rho_{6}(0,0,0,4,5,6)^{t r}+1=141$, $\rho_{6}(0,0,3,4,5,6)^{t r}+1=372, \rho_{6}(0,2,3,4,5,6)^{t r}+1=674$, and $\rho_{6}(1,2,3,4,5,6)^{t r}+1=877$, where these yield the 7 th row $\rho_{7}=(877,674,372,141,37,7,1)$.

\section{4. k-Peirce Matrix}

The Peirce matrix $\left[p_{i, j}\right](i, j \geq 0)$ was designed to generate Bell numbers.

$$
P=\left[p_{i, j}\right]=\left[\begin{array}{cccccc}
1 & & & & \\
1 & 2 & & & \\
2 & 3 & 5 & & \\
5 & 7 & 10 & 15 & \\
15 & 20 & 27 & 37 & 52
\end{array}\right]
$$

where it holds a recurrence $p_{i, j}=p_{i, j-1}+p_{i-1, j-1}$ with $p_{0,0}=$ $B_{0}, p_{n, 0}=p_{n-1, n-1}=B_{n}$. So the 6th row of $P$ begins with 52 followed by $67,87,114$, and 151 and then reach 203 , the next Bell number of 52 . Thus all Bell numbers are on both borders of $P$. The matrix is often called the Bell matrix or Aitken's array named after E. T. Bell and A. Aitken. Here we call it Peirce matrix after C. S. Peirce [10] to avoid confusion with the matrix of general Bell numbers in Section 3.

Now, for any $k \geq 1$, let $P^{(k)}=\left[p_{i, j}^{(k)}\right](i, j \geq 1)$ be a matrix satisfying the following two rules.

(i) Each $i$ th row begins with the Bell number $p_{i, 1}^{(k)}=B_{i-1}^{(0)}$. (ii)

$$
p_{i, j}^{(k)}=p_{i, j-1}^{(k)}+k p_{i-1, j-1}^{(k)} \quad \text { for all } i>1,
$$

and $p_{i, j}^{(k)}=0$ for $j>i$. 
Obviously $P^{(1)}$ is the Peirce matrix, and $P^{(k)}(k=2,3)$ are equal to

$$
\begin{aligned}
P^{(2)} & =\left[\begin{array}{llll}
1 & & & \\
1 & 3 & & \\
2 & 4 & 10 & \\
5 & 9 & 17 & 37
\end{array}\right] \\
\text { and } P^{(3)} & =\left[\begin{array}{llll}
1 & & & \\
1 & 4 & & \\
2 & 5 & 17 & \\
5 & 11 & 26 & 77
\end{array}\right]
\end{aligned}
$$

$$
\begin{aligned}
& 1 \\
& 1 \quad k+1 \\
& 2 \quad k+2 \quad k^{2}+2 k+2 \\
& 5 \quad 2 k+5 \quad k^{2}+4 k+5 \quad k^{3}+3 k^{2}+6 k+5 \\
& 15 \quad 5 k+15 \quad 2 k^{2}+10 k+15 \quad k^{3}+6 k^{2}+15 k+15 \quad k^{4}+4 k^{3}+12 k^{2}+20 k+15 \\
& 5215 k+525 k^{2}+30 k+522 k^{3}+15 k^{2}+45 k+52 k^{4}+8 k^{3}+30 k^{2}+60 k+52 \ldots
\end{aligned}
$$

When $k=1,2,3$, the r.diag. of $P^{(k)}$ yields $\{1,2,5,15, \cdots\}$, $\{1,3,10,37, \cdots\}$, and $\{1,4,17,77, \cdots\}$, where these correspond to $B^{(1)}, B^{(2)}$, and $B^{(3)}$.

Assume the r.diag

$$
\begin{aligned}
& \left\{1, k+1, k^{2}+2 k+2, k^{3}+3 k^{2}+6 k+5, k^{4}+4 k^{3}\right. \\
& \left.\quad+12 k^{2}+20 k+15, \cdots\right\}
\end{aligned}
$$

of $P^{(k)}$ equals the $k$-Bell numbers $B^{(k)}$. Then, due to (16), we have

$$
\left[\begin{array}{l}
B_{0}^{(k+1)} \\
B_{1}^{(k+1)} \\
B_{2}^{(k+1)} \\
B_{3}^{(k+1)} \\
B_{4}^{(k+1)}
\end{array}\right]=T\left[\begin{array}{c}
B_{0}^{(k)} \\
B_{1}^{(k)} \\
B_{2}^{(k)} \\
B_{3}^{(k)} \\
B_{4}^{(k)}
\end{array}\right]
$$

Notice that the left border is always comprised of Bell numbers, while the right diagonals (r.diag.) of $P^{(2)}$ and $P^{(3)}$ are of 2 and 3-Bell numbers, respectively. And the second right diagonal (2nd r.diag.) of $P^{(2)}$ equals the r.diag. of $P^{(3)}$. The next theorem shows that the r.diag. of $P^{(k)}=\left[p_{i, j}^{(k)}\right]$ $(k \geq 1)$ is composed of $k$-Bell numbers $B_{i}^{(k)}$.

Theorem 8. (1) The entries of r.diag. of $P^{(k)}$ are k-Bell numbers; i.e., $p_{i, i}^{(k)}=B_{i-1}^{(k)}$, while the entries of left border are Bell numbers.

(2) The 2nd r.diag. is composed of the $(k+1)$-Bell numbers; i.e., $p_{i, i-1}^{(k)}=B_{i}^{(k+1)}$.

Proof. Following the rules in (28), $P^{(k)}=\left[p_{i, j}^{(k)}\right]$ is equal to

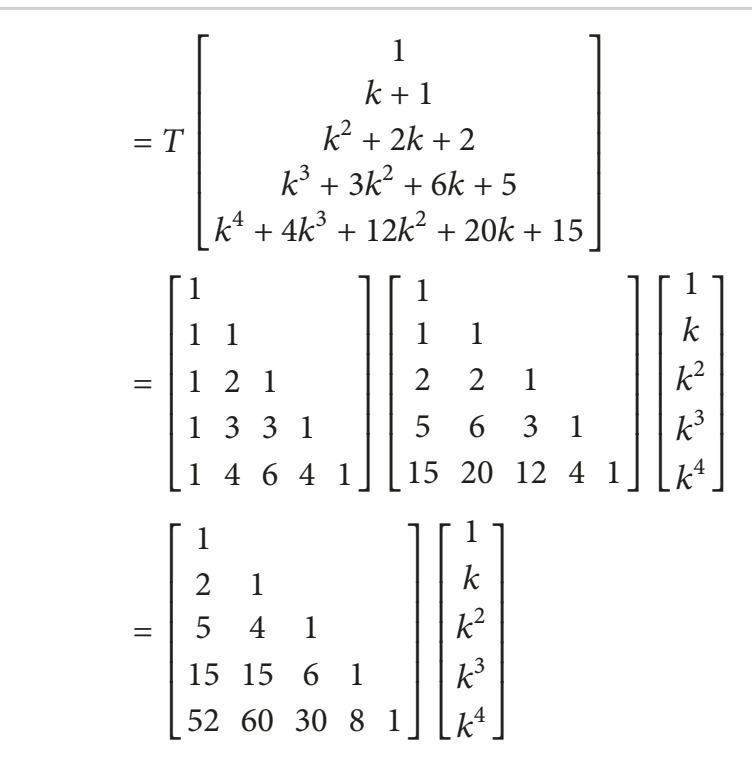

And it is not hard to see that it corresponds to the r.diag. of $P^{(k+1)}$

$$
\begin{aligned}
& 1 \\
& 1 \quad k+2 \\
& 2 k+3 \quad k^{2}+4 k+5 \\
& 5 \quad 2 k+7 \quad k^{2}+6 k+10 \quad k^{3}+6 k^{2}+15 k+15 \\
& 15 \quad 5 k+20 \quad 2 k^{2}+14 k+27 \quad k^{3}+9 k^{2}+30 k+37 \quad k^{4}+8 k^{3}+30 k^{2}+60 k+52 \\
& 5215 k+675 k^{2}+40 k+872 k^{3}+21 k^{2}+81 k+114
\end{aligned}
$$


Moreover, by comparing $P^{(k)}$ with $P^{(k+1)}$, we also notice that the r.diag. of $P^{(k+1)}$ equals the 2 nd r.diag. of $P^{(k)}$.

Owing to Theorem 8 , we may call $P^{(k)}$ the Peirce $k$ matrix. Write $\operatorname{diag}[a, b, c, \cdots]$ for the diagonal matrix having diagonal entries $a, b, c, \cdots$. Every $k$-Bell number can be obtained by Bell numbers as follows.

Theorem 9. $B_{n}^{(k)}=r_{n} \operatorname{diag}\left[B_{0}, B_{1}, \cdots, B_{n-1}\right]\left(k^{n-1}, \cdots, k, 1\right)^{t r}$.

Proof. When $k=1$, it is due to Theorem 1 . And, by Theorem 8, we have

$$
\begin{aligned}
{\left[\begin{array}{c}
B_{1}^{(k)} \\
B_{2}^{(k)} \\
B_{3}^{(k)} \\
B_{4}^{(k)} \\
B_{5}^{(k)}
\end{array}\right] } & {\left[\begin{array}{c}
1 \\
1+k \\
2+2 k+k^{2} \\
5+6 k+3 k^{2}+k^{3} \\
15+20 k+12 k^{2}+4 k^{3}+k^{4}
\end{array}\right] } \\
= & {\left[\begin{array}{cccc}
1 & & \\
1 & 1 & \\
2 & 2 & 1 & \\
5 & 6 & 3 & 1 \\
15 & 20 & 12 & 4
\end{array}\right]\left[\begin{array}{c}
1 \\
k \\
k^{2} \\
k^{3} \\
k^{4}
\end{array}\right] . }
\end{aligned}
$$

But we notice

$$
\begin{aligned}
& {\left[\begin{array}{ccccc}
1 & & & & \\
1 & 1 & & & \\
1 & 2 & 2 & & \\
1 & 3 & 6 & 5 & \\
1 & 4 & 12 & 20 & 15
\end{array}\right]} \\
& =\left[\begin{array}{llllll}
1 & & & & \\
1 & 1 & & & \\
1 & 2 & 1 & & \\
1 & 3 & 3 & 1 & \\
1 & 4 & 6 & 4 & 1
\end{array}\right]\left[\begin{array}{llllll}
1 & & & & \\
& 1 & & \\
& & 2 & & \\
& & & 5 & \\
& & & & 15
\end{array}\right] \\
& =T \operatorname{diag}\left[B_{0}, B_{1}, \cdots, B_{4}\right] \text {. }
\end{aligned}
$$

Hence, with the $n$th row $r_{n}$ of $T$, the $n$th $k$-Bell number $B_{n}^{(k)}$ is equal to $r_{n} \operatorname{diag}\left[B_{0}, \cdots, B_{n-1}\right]\left(k^{n-1}, \cdots, k, 1\right)^{t r}$.

For example, with $r_{6}=(1,5,10,10,5,1)$, the 6 th $k$-Bell number $B_{6}^{(k)}$ equals

$$
\begin{aligned}
r_{6} & \operatorname{diag}\left[B_{0}, \cdots, B_{5}\right]\left(k^{5}, \cdots, 1\right)^{\operatorname{tr}} \\
& =r_{6} \operatorname{diag}[1,1,2,5,15,52]\left(k^{5}, \cdots, 1\right)^{\operatorname{tr}} \\
& =k^{5}+5 k^{4}+20 k^{3}+50 k^{2}+75 k+52 .
\end{aligned}
$$

\section{Data Availability}

All data used to support the findings of this study are either obtained via the cited sources or included within the article.

\section{Conflicts of Interest}

The author declares that there are no conflicts of interest regarding the publication of this paper.

\section{Acknowledgments}

This work was supported by 2018 HanNam University Research Fund.

\section{References}

[1] A. Tucker, Applied combinatorics, John Wiley, New York, NY, USA, 2nd edition, 1984.

[2] P. Maltais and T. A. Gulliver, "Pascal matrices and Stirling numbers," Applied Mathematics Letters, vol. 11, no. 2, pp. 7-11, 1998.

[3] M. Z. Spivey and A. M. Zimmer, "Symmetric polynomials, Pascal matrices, and Stirling matrices," Linear Algebra and its Applications, vol. 428, no. 4, pp. 1127-1134, 2008.

[4] S.-L. Yang and H. You, "On a connection between the Pascal, Stirling and Vandermonde matrices," Discrete Applied Mathematics: The Journal of Combinatorial Algorithms, Informatics and Computational Sciences, vol. 155, no. 15, pp. 2025-2030, 2007.

[5] M. Abbas and S. Bouroubi, "On new identities for Bell's polynomials," Discrete Mathematics, vol. 293, no. 1-3, pp. 5-10, 2005.

[6] M. Griffiths, "Families of sequences from a class of multinomial sums," Journal of Integer Sequences, vol. 15, no. 1, Article 12.1.8, 10 pages, 2012.

[7] S. Noschese and P. E. Ricci, "Differentiation of multivariable composite functions and Bell polynomials," Journal of Computational Analysis and Applications, vol. 5, no. 3, pp. 333-340, 2003.

[8] J. Shallit, "A triangle for the Bell numbers," in A collection of manuscripts related to the Fibonacci sequence, pp. 69-71, Fibonacci Assoc., Santa Clara, Calif., 1980.

[9] I. Mezö, "TThe r-Bell numbers," Journal of Integer Sequences, vol. 14, no. 1, Article ID 11.1.1, 2011.

[10] C. S. Peirce, "On the algebra of logic," American Journal of Mathematics, vol. 3, no. 1, pp. 15-57, 1880. 


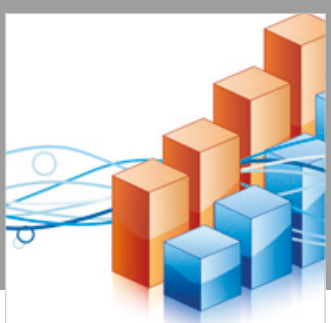

Advances in

Operations Research

\section{-n-m}
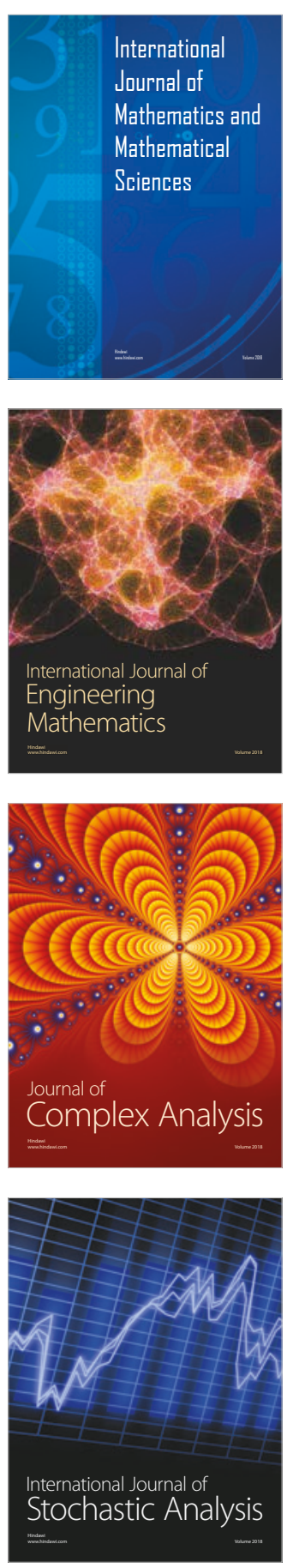
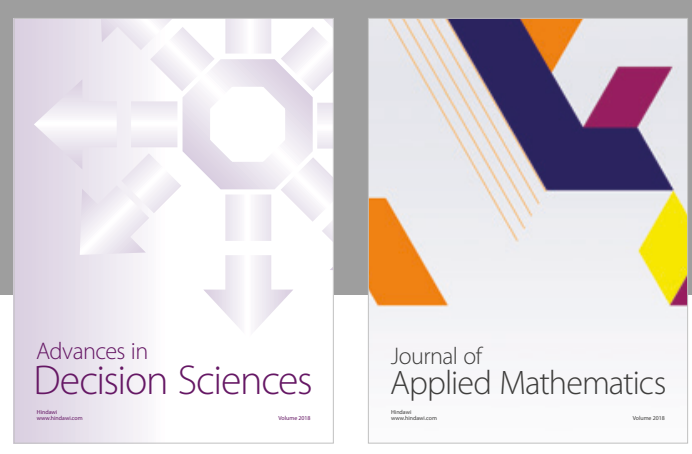

Journal of

Applied Mathematics
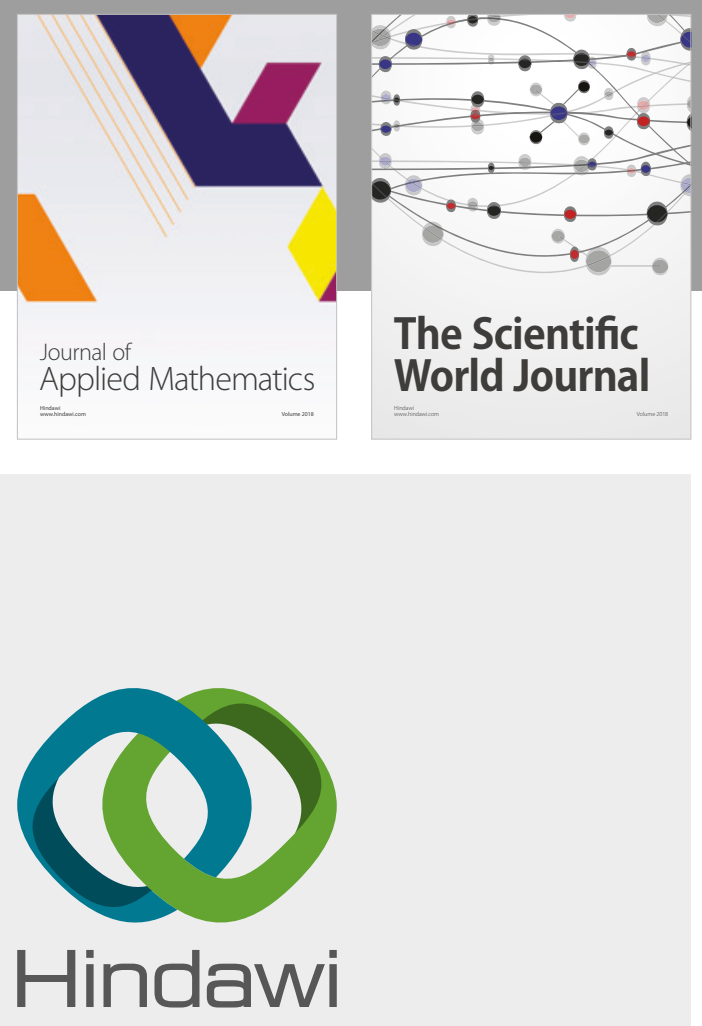

Submit your manuscripts at

www.hindawi.com

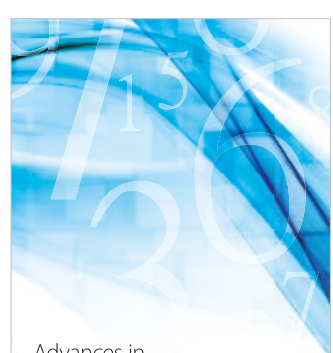

Advances in
Numerical Analysis
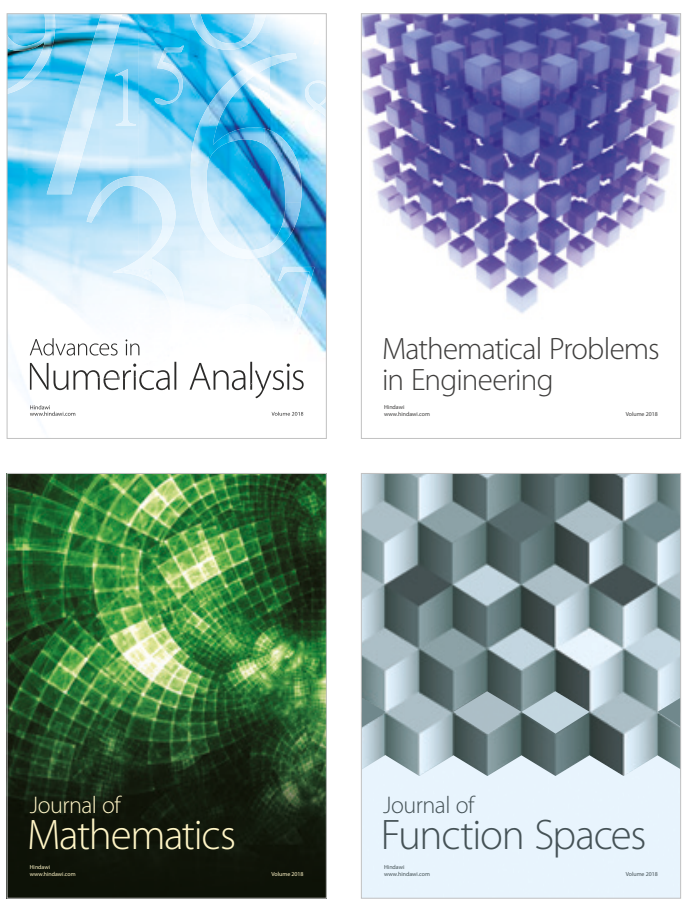

Mathematical Problems in Engineering

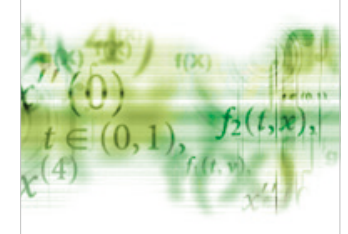

International Journal of

Differential Equations

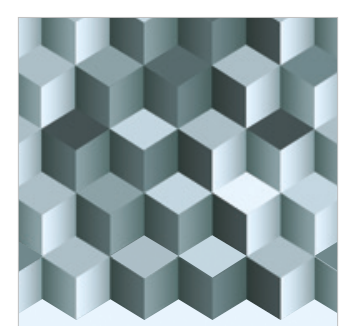

Journal of

Function Spaces

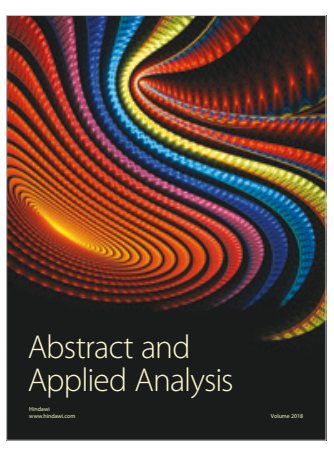

The Scientific

World Journal

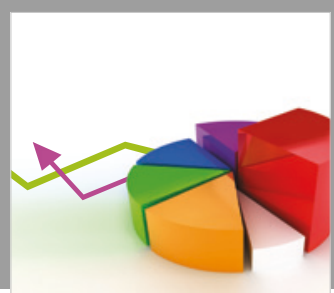

Journal of

Probability and Statistics
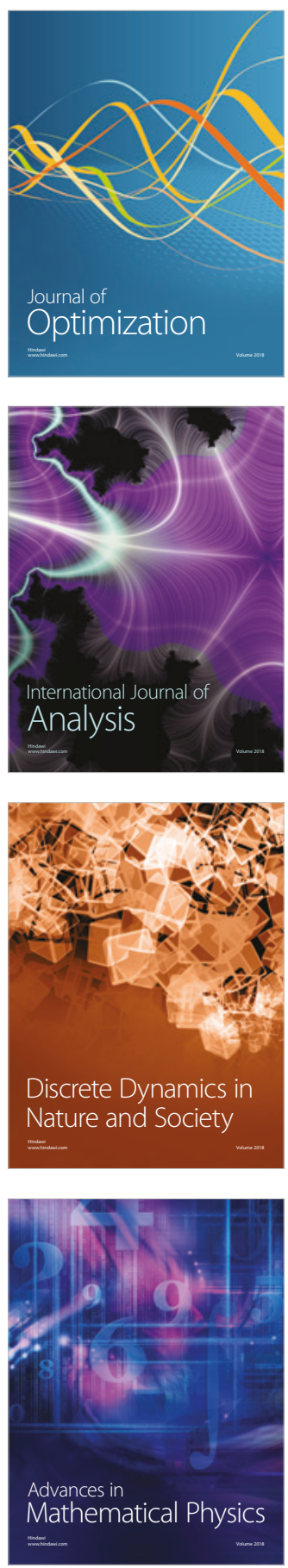